\title{
Existence and algorithm of solutions for a new system of generalized variational inclusions involving relaxed Lipschitz mappings
}

\author{
Rais Ahmad ${ }^{1}$
}

July 8, 2007

\begin{abstract}
We consider a new system for generalized variational inclusions in Hilbert spaces and define an iterative algorithm for finding the approximate solutions of this class of system of variational inclusions. We also establish that the approximate solutions obtained by our algorithm converge to the exact solution of new system of generalized variational inclusions. One can explore the role of our system of generalized variational inclusions for solving various known equilibrium problem and other related problems.
\end{abstract}

\section{Contents}

1 Introduction

See http://anziamj.austms.org.au/ojs/index.php/ANZIAMJ/article/view/55 for this article, (c) Austral. Mathematical Soc. 2007. Published July 8, 2007. ISSN 1446-8735 


\section{Introduction}

Because of applications in mechanics, physics, optimization and control, nonlinear programming, economics and engineering sciences, various variational inclusions have been intensively studied in recent years by Agarwal [1, 2], Ahmad et al. [5], Ding et al. [7] and Lee et al. [8]. Ansari and Yao [4] studied a system of variational inequalities using a fixed point theorem. Verma $[10,11,12]$ studied some systems of variational inequalities with single valued mappings and suggested some iterative algorithms to compute approximate solutions of these systems in Hilbert spaces. Agarwal [3] studied sensitivity analysis for a system of generalized nonlinear mixed quasi-variational inclusions with single-valued mappings. As an application of system of variational inclusions, Pang [9] showed that the traffic equilibrium problem, the Nash equilibrium problem, and the general equilibrium problem, can be modeled as a system of variational inequalities and inclusions.

Inspired and motivated by recent research work in this field, we introduce a new system of generalized variational inclusions and define an iterative algorithm. By the definition of relaxed Lipschitz mapping, we prove that the approximate solutions obtained by the iterative algorithm converge to the exact solution of our system of generalized variational inclusions. Our results improve and generalize many known corresponding results and can be used to solve different kinds of variational inequalities (inclusions), quasivariational inequalities (inclusions), complementarity problems, equilibrium 
problems and other related problems.

\section{Preliminaries}

Throughout, let $H$ be a real Hilbert space endowed with the inner product $\langle\cdot, \cdot\rangle$ and norm $\|\cdot\|$, respectively. Let $M, N: H \rightarrow 2^{H}$ be two maximal monotone mappings and $A, S, B, T, g: H \rightarrow H$ be nonlinear single valued mappings. We consider the problem of finding $x, y \in H$ such that

$$
\left.\begin{array}{l}
0 \in g(x)-g(y)+\rho(A(g(y))-S(y))+\rho M(g(x)), \\
0 \in g(y)-g(x)+\gamma(B(g(x))-T(x))+\gamma N(g(y)),
\end{array}\right\}
$$

which is called the system of generalized variational inclusions, where $\rho>0$ and $\gamma>0$ are two constants.

If $g=I$, the identity mapping, then the problem (1) reduces to the problem of finding $x, y \in H$ such that

$$
\left.\begin{array}{l}
0 \in x-y+\rho(A(y)-S(y))+\rho M(x), \\
0 \in y-x+\gamma(B(x)-T(x))+\gamma N(y) .
\end{array}\right\}
$$

A problem similar to (2) is considered by Agarwal et al. [3] and they discussed the sensitivity analysis of such a problem.

Definition 1 ([6]) If $M: H \rightarrow 2^{H}$ is a maximal monotone mapping, then for any fixed $\rho>0$, the mapping $J_{M}^{\rho}: H \rightarrow H$ defined by

$$
J_{M}^{\rho}(x)=(I+\rho M)^{-1}(x), \quad \text { for all } \quad x \in H,
$$

is said to be the resolvent operator of index $\rho$ of $M$, where $I$ is the identity mapping on $H$. Furthermore, the resolvent operator $J_{M}^{\rho}$ is single valued and nonexpansive, that is,

$$
\left\|J_{M}^{\rho}(x)-J_{M}^{\rho}(y)\right\| \leq\|x-y\|, \quad \text { for all } \quad x, y \in H .
$$




\section{Iterative algorithm}

First, we give the following lemma, the proof of which is a direct consequence of Definition 1 and hence is omitted.

Lemma $2 x, y \in H$ is the solution of system of generalized variational inclusions (1) if and only if it satisfies

$$
g(x)=J_{M}^{\rho}[g(y)-\rho(A(g(y))-S(y))], \quad \rho>0,
$$

where

$$
g(y)=J_{N}^{\gamma}[g(x)-\gamma(B(g(x))-T(x))], \quad \gamma>0 .
$$

The preceding lemma allows us to suggest the following iterative algorithm for system (1).

\section{Algorithm 1}

Given $x_{0} \in H$, compute $\left\{x_{n}\right\},\left\{y_{n}\right\}$ by the rule

$$
x_{n+1}=x_{n}-g\left(x_{n}\right)+J_{M}^{\rho}\left[g\left(y_{n}\right)-\rho\left(A\left(g\left(y_{n}\right)\right)-S\left(y_{n}\right)\right)\right], \quad \rho>0,
$$

where

$$
g\left(y_{n}\right)=J_{N}^{\gamma}\left[g\left(x_{n}\right)-\gamma\left(B\left(g\left(x_{n}\right)\right)-T\left(x_{n}\right)\right)\right], \quad \gamma>0, \quad n=0,1,2, \ldots .
$$

\section{Convergence theorem}

First, recall the following definitions. 
Definition 3 A mapping $g: H \rightarrow H$ is said to be

1. Strongly monotone, if there exists a constant $\delta>0$ such that

$$
\langle g(x)-g(y), x-y\rangle \geq \delta\|x-y\|^{2}, \quad \text { for all } \quad x, y \in H ;
$$

2. Lipschitz continuous, if there exists a constant $\lambda_{g}>0$ such that

$$
\|g(x)-g(y)\| \leq \lambda_{g}\|x-y\|, \quad \text { for all } \quad x, y \in H .
$$

Definition $4 A$ mapping $S: H \rightarrow H$ is said to be relaxed Lipschitz, if for a given $k \leq 0$,

$$
\langle S(x)-S(y), x-y\rangle \leq k\|x-y\|^{2}, \quad \text { for all } \quad x, y \in H .
$$

We apply Algorithm 1 to prove the following convergence theorem.

Theorem 5 Let $H$ be a real Hilbert space. Let $M, N: H \rightarrow 2^{H}$ be two maximal monotone mappings, $g: H \rightarrow H$ be strongly monotone with constant $\delta$ and Lipschitz continuous with constant $\lambda_{g}$. Let $A, S, B, T: H \rightarrow H$ be Lipschitz continuous mappings with Lipschitz constants $\lambda_{A}, \lambda_{S}, \lambda_{B}$ and $\lambda_{T}$, respectively and let $S$ be relaxed Lipschitz with constant $k$. If there exists constants $\rho>0$ and $\gamma>0$ such that

$$
\begin{aligned}
& \left|\rho+\frac{\left(C-\theta_{1}\right) \lambda_{A} \lambda_{g}-k}{\lambda_{S}^{2}-\lambda_{A}^{2} \lambda_{g}^{2}}\right| \\
& <\frac{\sqrt{\left[\left(C-\theta_{1}\right) \lambda_{A} \lambda_{g}-k\right]^{2}-\left(\lambda_{S}^{2}-\lambda_{A}^{2} \lambda_{g}^{2}\right)\left[1-\left(C-\theta_{1}\right)^{2}\right]}}{\lambda_{S}^{2}-\lambda_{A}^{2} \lambda_{g}^{2}},
\end{aligned}
$$

where

$$
\left(\left(C-\theta_{1}\right) \lambda_{A} \lambda_{g}-k\right)^{2}>\left(\lambda_{S}^{2}-\lambda_{A}^{2} \lambda_{g}^{2}\right)\left(1-\left(C-\theta_{1}\right)^{2}\right) \quad \text { and } \quad \lambda_{S}>\lambda_{A} \lambda_{g},
$$

where $C=\delta\left(1-\theta_{1}\right) / \theta_{3}, \theta_{1}=\sqrt{1-2 \delta+\lambda_{g}^{2}}, \theta_{3}=\left[\lambda_{g}+\gamma \lambda_{B} \lambda_{g}+\gamma \lambda_{T}\right]$, then the iterative sequences $\left\{x_{n}\right\},\left\{y_{n}\right\}$ generated by Algorithm 1 strongly converge to $x, y$, respectively, in $H$, and $x, y \in H$ is a solution of system of generalized variational inclusions (1). 
Proof: From Algorithm 1 and using the nonexpansiveness of resolvent operator, we have

$$
\begin{aligned}
& \left\|x_{n+1}-x_{n}\right\|=\| x_{n}-g\left(x_{n}\right)+J_{M}^{\rho}\left[g\left(y_{n}\right)-\rho\left(A\left(g\left(y_{n}\right)\right)-S\left(y_{n}\right)\right]\right. \\
& -\left[x_{n-1}-g\left(x_{n-1}\right)+J_{M}^{\rho}\left[g\left(y_{n-1}\right)-\rho\left(A\left(g\left(y_{n-1}\right)-S\left(y_{n-1}\right)\right]\right] \|\right.\right. \\
& \leq\left\|x_{n}-x_{n-1}-\left(g\left(x_{n}\right)-g\left(x_{n-1}\right)\right)\right\|+\| g\left(y_{n}\right)-g\left(y_{n-1}\right)-\rho\left(A \left(g\left(y_{n}\right)\right.\right. \\
& \left.-A\left(g\left(y_{n-1}\right)\right)\right)+\rho\left(S\left(y_{n}\right)-S\left(y_{n-1}\right)\right) \| \\
& \leq\left\|x_{n}-x_{n-1}-\left(g\left(x_{n}\right)-g\left(x_{n-1}\right)\right)\right\|+\left\|y_{n}-y_{n-1}-\left(g\left(y_{n}\right)-g\left(y_{n-1}\right)\right)\right\| \\
& +\left\|y_{n}-y_{n-1}+\rho\left(S\left(y_{n}\right)-S\left(y_{n-1}\right)\right)\right\|+\rho\left\|A\left(g\left(y_{n}\right)\right)-A\left(g\left(y_{n-1}\right)\right)\right\| .(8)
\end{aligned}
$$

By Lipschitz continuity and strong monotonicity of $g$, we obtain

$$
\left\|x_{n}-x_{n-1}-\left(g\left(x_{n}\right)-g\left(x_{n-1}\right)\right)\right\|^{2} \leq\left(1-2 \delta+\lambda_{g}^{2}\right)\left\|x_{n}-x_{n-1}\right\|^{2},
$$

and

$$
\left\|y_{n}-y_{n-1}-\left(g\left(y_{n}\right)-g\left(y_{n-1}\right)\right)\right\|^{2} \leq\left(1-2 \delta+\lambda_{g}^{2}\right)\left\|y_{n}-y_{n-1}\right\|^{2} .
$$

Since $S$ is Lipschitz continuous and relaxed Lipschitz, we have

$$
\begin{aligned}
& \left\|y_{n}-y_{n-1}+\rho\left(S\left(y_{n}\right)-S\left(y_{n-1}\right)\right)\right\|^{2} \\
& =\left\|y_{n}-y_{n-1}\right\|^{2}+2 \rho\left\langle S\left(y_{n}\right)-S\left(y_{n-1}\right), y_{n}-y_{n-1}\right\rangle \\
& \quad+\rho^{2}\left\|S\left(y_{n}\right)-S\left(y_{n-1}\right)\right\|^{2} \\
& \leq \quad\left\|y_{n}-y_{n-1}\right\|^{2}+2 \rho k\left\|y_{n}-y_{n-1}\right\|^{2}+\rho^{2} \lambda_{S}^{2}\left\|y_{n}-y_{n-1}\right\|^{2} \\
& \quad=\left(1-2 \rho k+\rho^{2} \lambda_{S}^{2}\right)\left\|y_{n}-y_{n-1}\right\|^{2} .
\end{aligned}
$$

By the Lipschitz continuity of $A$ and $g$ we have

$$
\left\|A\left(g\left(y_{n}\right)\right)-A\left(g\left(y_{n-1}\right)\right)\right\| \leq \lambda_{A} \lambda_{g}\left\|y_{n}-y_{n-1}\right\|,
$$

using (9)-(12), (8) becomes

$$
\left\|x_{n+1}-x_{n}\right\| \leq \sqrt{1-2 \delta+\lambda_{g}^{2}}\left\|x_{n}-x_{n-1}\right\|
$$




$$
+\left(\sqrt{1-2 \delta+\lambda_{g}^{2}}+\sqrt{1-2 \rho k+\rho^{2} \lambda_{S}^{2}}+\rho \lambda_{A} \lambda_{g}\right)\left\|y_{n}-y_{n-1}\right\|
$$

Now, we have

$\left\|g\left(y_{n}\right)-g\left(y_{n-1}\right)\right\|\left\|y_{n}-y_{n-1}\right\| \geq\left\langle g\left(y_{n}\right)-g\left(y_{n-1}\right), y_{n}-y_{n-1}\right\rangle \geq \delta\left\|y_{n}-y_{n-1}\right\|^{2}$, which implies

$$
\begin{aligned}
&\left\|y_{n}-y_{n-1}\right\| \leq \frac{1}{\delta}\left\|g\left(y_{n}\right)-g\left(y_{n-1}\right)\right\| \\
& \leq \frac{1}{\delta} \| J_{N}^{\gamma}\left[g\left(x_{n}\right)-\gamma\left(B\left(g\left(x_{n}\right)\right)-T\left(x_{n}\right)\right)\right]-J_{N}^{\gamma}\left[g\left(x_{n-1}\right)\right. \\
&\left.\quad-\gamma\left(B\left(g\left(x_{n-1}\right)\right)-T\left(x_{n-1}\right)\right)\right] \| \\
& \leq \frac{1}{\delta} \| g\left(x_{n}\right)-g\left(x_{n-1}\right)-\gamma\left(B\left(g\left(x_{n}\right)\right)-\left(B\left(g\left(x_{n-1}\right)\right)\right)\right. \\
& \quad+\gamma\left(T\left(x_{n}\right)-T\left(x_{n-1}\right)\right) \| \\
& \leq \frac{1}{\delta}\left[\left\|g\left(x_{n}\right)-g\left(x_{n-1}\right)\right\|+\gamma \|\left(B\left(g\left(x_{n}\right)\right)-\left(B\left(g\left(x_{n-1}\right)\right) \|\right.\right.\right. \\
&\left.\quad+\gamma\left\|T\left(x_{n}\right)-T\left(x_{n-1}\right)\right\|\right] \\
& \leq \frac{1}{\delta}\left[\lambda_{g}+\gamma \lambda_{B} \lambda_{g}+\gamma \lambda_{T}\right]\left\|x_{n}-x_{n-1}\right\| .
\end{aligned}
$$

Combining (13) and (14), we have

$$
\left\|x_{n+1}-x_{n}\right\| \leq\left(\theta_{1}+\left(\theta_{1}+\theta_{2}\right) \frac{1}{\delta} \theta_{3}\right)\left\|x_{n}-x_{n-1}\right\|,
$$

where $\theta_{1}=\sqrt{1-2 \delta+\lambda_{g}^{2}}, \theta_{2}=\sqrt{1-2 \rho k+\rho^{2} \lambda_{s}^{2}}+\rho \lambda_{A} \lambda_{g}, \theta_{3}=\left[\lambda_{g}+\right.$ $\left.\gamma \lambda_{B} \lambda_{g}+\gamma \lambda_{T}\right]$.

Hence, we have

$$
\left\|x_{n+1}-x_{n}\right\| \leq \theta\left\|x_{n}-x_{n-1}\right\|
$$

where $\theta=\theta_{1}+\left(\theta_{1}+\theta_{2}\right) \theta_{3} / \delta$. By Condition $(7)$, we have $\theta<1$. 
Inequality (16) implies that $\left\{x_{n}\right\}$ is a Cauchy sequence. Also, (14) implies that $\left\{y_{n}\right\}$ is a Cauchy sequence in $H$. Hence, there exist $x, y \in H$ such that $x_{n} \rightarrow x$ and $y_{n} \rightarrow y$. Since $g, A, S, B, T, J_{M}^{\rho}$ and $J_{N}^{\gamma}$ are continuous, then it follows from Algorithm 1 that $x, y \in H$ satisfy (3), (4) and thus by Lemma 2, it follows that $x, y \in H$ is a solution of system of generalized variational inclusions (1). This completes the proof.

\section{References}

[1] R. P. Agarwal, Y. J. Cho and N. J. Huang, Sensitivity analysis for strongly nonlinear quasi-variational inclusions, Appl. Math. Lett. 13(6), 19-24, (2000). E2

[2] R. P. Agarwal, N. J. Huang and Y. J. Cho, Generalized nonlinear mixed implicit quasi-variational inclusions with set-valued mappings, J. Inequal. Appl. 7(6), 807-828, (2002). E2

[3] R. P. Agarwal, N. J. Huang and M. Y. Tan, Sensitivity analysis for a new system of generalized nonlinear mixed quasi-variational inclusions, Appl. Math. Lett. 17, 345-352, (2004). E2, E3

[4] Q. H. Ansari and J. C. Yao, A fixed point theorem and its applications to a system of variational inequalities, Bull. Austral. Math. Soc. 59(3), 433-442, (1999). E2

[5] R. Ahmad and Q. H. Ansari, An iterative algorithm for generalized nonlinear variational inclusions, Appl. Math. Lett. 13(5), 23-26, (2000). E2

[6] J. P. Aubin and I. Ekeland, Applied Nonlinear Analysis, John Wiley and Sons, New York, 1984. E3 
[7] X. P. Ding and C. L. Luo, Perturbed proximal point algorithms for general quasi-variational-like inclusions, J. Comput. Appl. Math. 113, 153-165, (2000). E2

[8] C. H. Lee, Q. H. Ansari and J. C. Yao, A perturbed algorithm for strongly nonlinear variational inclusions, Bull. Austral. Math. Soc. 62, 417-426, (2000). E2

[9] J. S. Pang, Asymmetric variational inequality problem over product of sets:Applications and iterative methods, Math Prog. 31, 206-219, (1985). E2

[10] R. U. Verma, Partially relaxed monotone mappings and a new system of nonlinear variational inequalities, Nonlinear Funct. Anal. Appl. 5(1), 65-72, (2000). E2

[11] R. U. Verma, Projection method, Algorithm and a new system of nonlinear variational inequalities, Comput. Math. Appl. 41(7-8), 1025-1031, (2001). E2

[12] R. U. Verma, Generalized system for relaxed cocoercive variational inequalities and projection methods, J. Optim. Theory Appl. 121(1), 203-210, (2004). E2 


\section{Author address}

1. Rais Ahmad, Department of Mathematics, Aligarh Muslim University, Aligarh-202002, India mailto:raisain@lycos.com 